\title{
On jet instability modes of a subsonic Hartmann whistle
}

\author{
RAMANATHAN VARADHARAJAN ${ }^{1,2, *}{ }^{-}$, MANU KAMIN $^{1,3}$, SUBRAMANIAN GANESH $^{1}$ and \\ JOSEPH MATHEW ${ }^{1}$ \\ ${ }^{1}$ Department of Aerospace Engineering, Indian Institute of Science, Bangalore 560012, India \\ ${ }^{2}$ Present Address: Physical Chemistry and Soft Matter, Wageningen University and Research Center, \\ 6708 WE Wageningen, The Netherlands \\ ${ }^{3}$ Present Address: Aerospace Engineering and Engineering Mechanics, University of Cincinnati, Cincinnati, \\ OH 45221, USA \\ e-mail: ramanathan.varadharajan@wur.nl
}

MS received 3 March 2017; revised 4 February 2018; accepted 15 February 2018; published online 17 July 2018

\begin{abstract}
Numerical experiments to understand the resonant acoustic response of a subsonic jet impinging on the mouth of a tube, known as the Hartmann whistle configuration, were performed as large-eddy simulations. The tube length was chosen so that its fundamental duct mode, for one end closed and one end open, would match the dominant mode in the exciting jet. When the tube mouth was placed in the path of a regular stream of vortex rings, formed by the instability of the jet's bounding shear layer, a strong resonant, tonal response (whistling) was obtained. At three diameters from the jet, OASPL was 150-160 dB. A tube with a thicker lip generated a louder response. When the tube was held closer to the nozzle exit, the impinging unsteady shear layer could not provoke any significant resonance. The simulations reveal that the tonal response of a Hartmann whistle operating in subsonic mode is significant.
\end{abstract}

Keywords. Hartmann whistle; jet-instability mode; subsonic flow; large-eddy simulation.

\section{Introduction}

A Hartmann whistle is a tube with one closed end, placed with the open end facing a free jet. In most studies the jet has been supersonic, including the one that led to Hartmann's discovery [1]. Often the jets have been sonic or at underexpanded supersonic conditions at the nozzle exit plane. High-intensity, narrow-spectrum sound (a whistle) is produced, and rapid heating of the gas is observed inside the tube, near its closed end. Whistling occurs for a wide range of pressure ratios, tube lengths and stand-off distances. A comprehensive, recent review is available [2]. Three modes have been identified, namely, the screech mode, the jet regurgitant mode and the jet instability mode. The screech and regurgitant modes are observed in supersonic jets, and the response to subsonic jets has been termed the instability mode. Whistles excited by supersonic jets have found various applications (industrial whistles, acoustic actuators, musical instruments, etc.), and have been the subject of many studies [3-8]. In these, numerical solutions were obtained from two-dimensional RANS models [4, 6] only. Here we report results of numerical experiments using a large-eddy simulation (LES) model (3-

\footnotetext{
*For correspondence
}

d, unsteady) to understand the flow behaviour of a Hartmann whistle configuration excited by subsonic jets.

In many of the earlier experiments, tripping was considered essential to obtaining a strong response. These developments are discussed in the review by Raman and Srinivasan [2]. In supersonic streams, a ring or a wedge has served as a trip. By placing a thin nylon thread (diameter $10^{-3} \mathrm{~cm}$ ) across the jet axis, Sprenger [9] was able to produce oscillating flow with low-Mach-number $(M=0.52)$ subsonic jets. Brocher et al [10] obtained a strong response even at $M=0.12$ and suggested that it is essential to reduce the energy of the jet in the neighbourhood of its axis. They placed a thin cylinder axially in the jet nozzle, and produced a wake in the central region of the jet (region of lower mean kinetic energy). However, rather than any energetics processes, all these configurations cause vortex shedding that forces resonant oscillations in the tube. Borcher et al [10] proceeded to obtain theoretical relations for maximum amplitude and frequency for subsonic flows. Theoretical models to understand the oscillation mechanism were based on either wave or sound-speed diagrams, assuming isentropic, inviscid flow. Flow in the jet was assumed to be quasi-steady. In later experiments, Sarohia and Back [11] observed that the oscillations in the tube occur at the vortex-shedding frequency. 
Brocher and Duport [12] conducted experiments with resonance tubes mounted in a subsonic tunnel so that the upstream flow was uniform, and not that of a jet. Prismatic (wedge and square cross-section) bodies were mounted close to the tube mouth to trip the flow. The distance between the rear end of these trips and the tube mouth was varied from 10 to $34 \mathrm{~mm}$. Tubes had a square cross-section of side $36 \mathrm{~mm}$, and lengths 200 and $355 \mathrm{~mm}$. The pressure at the closed end of the tube was measured. Below a critical velocity of the incident flow, pressure oscillations were small and irregular. As the critical velocity was approached, the pressure oscillation amplitude increased rapidly. They discuss the connection to organ pipe response, where, also, no sound is emitted below a critical velocity of the air stream that impinges on the pipe mouth. They argued that the strong, resonant response was a coupling between an instability wave in the shear layer separating from the tripping device and the standing acoustic wave in the tube. They concluded that 'the device constitutes a very simple, powerful, sound source' for use in applications or research [12].

Clearly, detailed, time-accurate, numerical simulations, which are now possible, can provide a better understanding of the mechanisms, especially the effects of ad hoc designs, like wires and stems, for enhancing the response.

Owing to the well-known strong response in supersonic streams, the subsonic whistle has not received much attention. The focus of our studies was to determine the response of a Hartmann whistle-like configuration placed in a subsonic stream. The whistle is then excited by the jet instability mode. Two qualitatively different types of responses were obtained by placing the tube at two locations in a subsonic jet-close to the nozzle exit where the jet's bounding shear layer experiences instability and provides an unsteady excitation at the tube mouth, and secondly, further downstream where the excitation is by a train of vortex rings that have developed from the shear layer. A strong resonant response was found in the latter case. This is consistent with conclusions on whistle designs that incorporated stems or wires to promote vortex shedding that have been discussed earlier. The paper begins with the numerical method for LES and a relevant validation with a round jet, followed by results of whistle simulations in the two configurations.

\section{Numerical method}

The flows considered are governed by the Navier-Stokes equations for compressible flow:

$$
\begin{gathered}
\frac{\partial \rho}{\partial t}+\frac{\partial \rho u_{i}}{\partial x_{i}}=0 \\
\frac{\partial \rho u_{i}}{\partial t}+\frac{\partial \rho u_{i} u_{j}}{\partial x_{j}}=-\frac{\partial p}{\partial x_{i}}+\frac{\partial \tau_{i j}}{\partial x_{j}}
\end{gathered}
$$

$$
\frac{\partial \rho E}{\partial t}+\frac{\partial}{\partial x_{j}}\left[(\rho E+p) u_{i}\right]=\frac{\partial q_{i}}{\partial x_{i}}+\frac{\partial u_{j} \tau_{i j}}{\partial x_{i}} .
$$

Here $\rho$ is the density, $u_{i}$ are Cartesian velocity components, $p$ is pressure, $E$ is the energy, $\rho E=\left(\rho u_{k} u_{k}\right) / 2+p /(\gamma-1)$ and $q_{i}$ is the heat flux. The Prandtl number was set to a constant value of 0.71 . Shear stress is

$$
\tau_{i j}=\mu\left(\frac{\partial u_{i}}{\partial x_{j}}+\frac{\partial u_{j}}{\partial x_{i}}-\frac{2}{3}\left[\frac{\partial u_{k}}{\partial x_{k}}\right] \delta_{i j}\right),
$$

where $\mu$, the dynamic viscosity, was calculated from Sutherland's law

$$
\mu=\frac{1.458 T^{1.5}}{(T+110.0)}
$$

Since the flows considered are not laminar, the numerical solution was found as an LES using the explicit filtering method of Mathew et al [13]. Essential requirements for this method are the use of a high-resolution numerical method along with a high-resolution, low-pass spatial filter applied to transported variables after every time step. This approach to LES has been used successfully, for several types of flows, by at least two other groups $[14,15]$. Here, a Cartesian grid was used with an 8thorder compact difference formula for spatial derivatives, split into a forward and a backward step, extending the method of Hixon and Turkel [16]. Time-stepping was by a 2nd-order Runge-Kutta scheme. A one-parameter fourth-order compact filter [17] was applied with filter parameter $\alpha=0.475$ in the stream-wise direction and 0.498 in the cross-stream directions. Partially non-reflecting, characteristic boundary conditions were specified at boundaries [18]. A constant time-step estimated from a maximum CFL number of 0.075 was used. The numerical method including boundary treatments, combining non-reflecting conditions, stretched grid buffer zone and anchoring inflow variables to target values was developed and tested for jet aeroacoustics studies. Ganesh [19], who developed the initial version of this code, provides a detailed discussion.

\subsection{Validation}

LES of a subsonic round jet is provided as a relevant validation before performing whistle simulations. The method (and core components of the code) has also been used for wall-bounded flows such as impinging flow [19]. The whistle simulations were performed using the same round jet with a tube placed downstream. Hence, the accurate simulation of the round jet also provides guidelines for setting up the whistle problem. The round jet of mean radius $r_{j}$ was defined by the radial profile of its axial velocity at the inflow plane. Its mean value 


$$
U=\frac{U_{j}}{2}+\frac{U_{j}}{2} \tanh \left(\frac{r_{j}-r}{2 \delta_{2}}\right)
$$

defines a jet of roughly uniform velocity $U_{j}$ bounded by an annular shear layer, and emerging into a quiescent region; shear layer momentum thickness $\delta_{2}=r_{j} / 20$ [20]. Mean inlet pressure $p_{j}=1823.85 \mathrm{~Pa}$. Inlet density is determined using the Crocco-Busemann relationship. Ambient density $\rho_{a}=$ $0.022 \mathrm{~kg} / \mathrm{m}^{3}$ and inflow density $\rho_{j}=0.02564 \mathrm{~kg} / \mathrm{m}^{3}$.

To induce breakdown to turbulence, fluctuations are added to the axial velocity in the shear layer as

$$
\begin{aligned}
& u^{\prime}(\theta, t)=\frac{U_{j}}{1000} A \\
& {\left[\sum_{m=1}^{6} \sin \left(m \theta+\alpha_{m}+2 \pi f_{m} t\right)+\sum_{m=7}^{8} \sin \left(\psi_{m} t\right)\right]}
\end{aligned}
$$

with

$$
A=\exp \left(-128\left[\frac{r-R}{R}\right]^{2}\right)
$$

Here $\theta$ is the azimuthal angle, $f_{m}$ are frequencies with random phases $\alpha_{m}$ and $R=0.875 r_{j}$. The frequencies correspond to Strouhal numbers $\mathrm{St}=2 f r_{j} / U_{j}=0.2,0.27,0.31,0.39,0.45$ and 0.53, as in Bodony and Lele [20]. The perturbation $u^{\prime}$ was added at both stages of the time-stepping.
Simulations were performed on multiple grids and at different Reynolds numbers. Here we show results of a case where the Reynolds number based on reference quantities from the jet at the inflow $\operatorname{Re}=2 \rho_{j} U_{j} r_{j} / \mu_{j}=88,000$, corresponding to an aeroacoustics reference case. In the following, all lengths are scaled with the nominal jet radius $r_{j}$. There were $386 \times 201 \times 201$ points in the axial and transverse directions on a Cartesian grid on the domain of extent $0<x<100$ and $-16<y, z<16$. The grid spacing is uniform in the near field and is then stretched. In the crossstream directions, grid spacing $\Delta y=\Delta z=1 / 10$ for $0<|y|,|z|<10$. Beyond this there is $1.5 \%$ stretching in geometric progression. In the axial direction $\Delta x=0.25 \Delta y$ for $0<x<75$. Then there is $2 \%$ stretching till 90 and $5 \%$ beyond till the outflow plane; the latter region serves as a buffer zone where, due to the aggressive stretching, smallscale structures are diffused.

Figure 1a is a visualization of the jet with contours of vorticity magnitude at an instant. The roll-up of the initial annular shear layer into vortex rings and sudden breakdown around $x \approx 12$ terminating the potential core are evident. Further downstream, the flow is turbulent and becomes selfpreserving.

Figure $1 \mathrm{~b}$ shows the decay of the centreline velocity $U_{c}$. The reciprocal of $U_{c}$ is expected to grow linearly in selfpreserving round jets. This is a sensitive test of LES. It is our experience with several types of coarse-grid
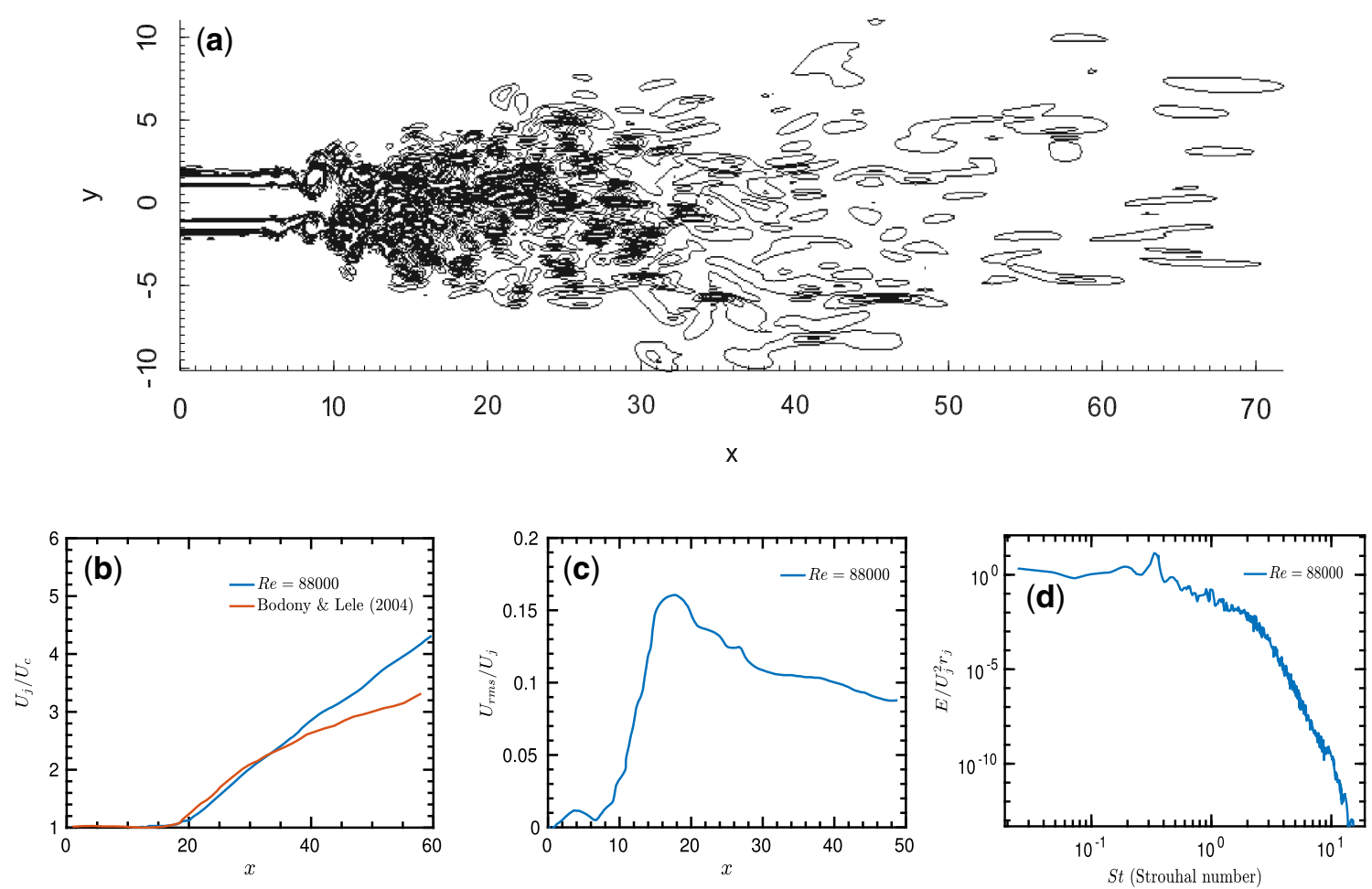

Figure 1. (a) Contours of vorticity magnitude on the plane $z=0$ at an instant. (b) Centreline velocity decay. (c) Turbulent fluctuation development for the round jet simulations. (d) Turbulent kinetic energy spectrum at $x=14$. A dominant mode occurs at $\mathrm{St}=0.334$ (for the results presented, $M=0.9$ and $\operatorname{Re}=88000$ ). 
simulations that when the scale range simulated is not adequate, a parabola-like curve rather than a straight line is obtained. The slope of the straight line $B=6.28$ is comparable to the value 6.06 obtained in the benchmark experiments of Panchapakesan and Lumley [22] for a lowspeed jet at $\operatorname{Re}=11000$. The slightly larger value is expected as jet Mach number is increased, as in our case [23]. The close agreement with another simulation [20], which was performed over a shorter length (useful region $x<30$ ), is evident. The development of turbulent fluctuations also agrees well with previous simulations, see figure 1c. Results of simulations for $\operatorname{Re}=3,600$ and 400,000 are available [24, 25]. A dominant frequency can be identified in the spectra of signals taken near the end of the potential core with a Strouhal number $\mathrm{St}=0.334$ (figure 1d); the reported range is $0.3-0.4$ [21, 26]. For the whistle simulations, the tube length will be chosen so that the duct mode frequency is close to that of this jet instability response.

\section{Hartmann whistle studies}

For the Hartmann whistle configurations, the exciting round jet was taken to be that in the validation discussed earlier. The grid spacing in this region was identical, but the Reynolds number was lower at 16,000 and the Mach number was 0.3 at inflow. Thin annular shear layers can be expected to have stability characteristics similar to those of plane shear layers. The perturbations added to the shear layer at inflow have Strouhal numbers $\mathrm{St}=0.2,0.27,0.31$, $0.39,0.45$ and 0.53 , as in the free jet simulation described earlier and in Bodony and Lele [20]. In tubes that are not too short, standing acoustic modes can be excited. In a tube of length $L$, with one closed end and one open end, the fundamental frequency is

$$
f=\frac{a}{4 L}
$$

where $a$ is the sound speed. In one configuration the tube was placed just downstream of where the jet shear layer had rolled up into vortex rings but the potential core had not broken down. Since the free jet exhibited a mode with $\mathrm{St}=0.34$, the tube length $L$ was chosen so that the duct mode was at $\mathrm{St}=0.33$. Distance between jet inflow plane and tube opening, the stand-off distance, scaled with jet radius $r_{j}$ is $S=20$. This is labelled as the $\mathrm{S} 1$ simulation. In the second simulation (S2), the tube was placed upstream $S=7.5$, where the annular shear layer had not broken up into distinct vortex rings; duct length was chosen so that the duct mode is at $\mathrm{St}=0.31$, which is the dominant response of the initial shear layer. We can then expect resonant, large amplitude, excitation of the duct-whistling.

A longitudinal section of the computational domain is shown in figure $2 \mathrm{a}$. A round jet of unit radius enters the domain through the plane $x=0$. Flow was simulated in the region $0<x<50$ and $-16<y, z<16$. In the cross-stream directions, grid spacing $\Delta y=\Delta z=1 / 10$ in $0<|y|,|z|<10$; beyond this, grid spacing is increased at $1.5 \%$ in geometric progression. In the axial direction, $\Delta x=0.25 \Delta y$ for $0<x<40$. Then there is $2 \%$ stretching till $x=45$, and $5 \%$ beyond till the outflow plane. A tube with a square crosssection, closed at one end, was placed with tube axis on the jet axis as shown. Tube walls coincided with grid points. For computational efficiency, the computational domain was divided into 3 blocks. The discretized equations were solved to advance the state at interior points in each block in parallel using MPI. Within each block, code segments were executed in parallel using OpenMP. At block interfaces, grids overlap. At the solid boundaries of the tube, one-sided differences were used. Pressure time series were collected at several points. Signals used in the analyses are for 10 flow-through times; flow-through time was estimated as the duration for a particle to travel from nozzle exit to tube end with particle velocity falling linearly. There were $224 \times 201 \times 201$ points in the axial and transverse directions, but of these, no calculations were done at points within the tube walls. No-slip boundary conditions were enforced at tube walls.

Dimensions of the tubes in the simulations are listed in table $1 . \mathrm{T} 2$ is a tube with twice the wall or lip thickness compared with $\mathrm{T} 1$, and tube $\mathrm{T} 3$ has increased inlet area. The four simulation cases are listed in table 2, with labels that are a combination of tube type and duct mode.

\subsection{Tube excited by ring vortices}

The flowfield at an instant for case S1T1 is shown in figure $2 b$ with contours of the velocity magnitude on the midplane $z=0$. The open end of the tube was at $x=20$. The impinging jet is deflected by the tube. Inflow perturbations grow on the shear layer and roll up into vortex rings. These rings impinge on the jet and expand rapidly around the tube. Time series of pressure inside the tube at the closed end $(x=24.5, y=z=0)$ and near the tube lip $(x=20, y= \pm 2.25, z=0)$ are shown in figure $2 \mathrm{c}$. The amplitude of oscillations is larger at the closed end (cavity end wall), and more regular; it is more broadband near the tube lip (top/bottom - shedding region). Figure 3a shows spectra at these same locations. The inset figure shows the tonal components more clearly; the dominant tone is at $\mathrm{St}=0.33$. The simulation with a tube with thicker lip, S1T2, is qualitatively similar. The spectra are shown in figure $3 \mathrm{~b}$. The dominant frequency excited is still $\mathrm{St}=0.33$ as can be expected for a resonant duct mode, and the amplitude is about the same. There are other tones of smaller amplitudes; those at lower frequencies have relatively larger amplitudes. These tones are not harmonics or sub-harmonics and do not represent waveform distortion. We shall refer to these as secondary modes. The lower frequency secondary modes have a larger amplitude with the thicker tube wall. It should also be noted that the power 
(a)

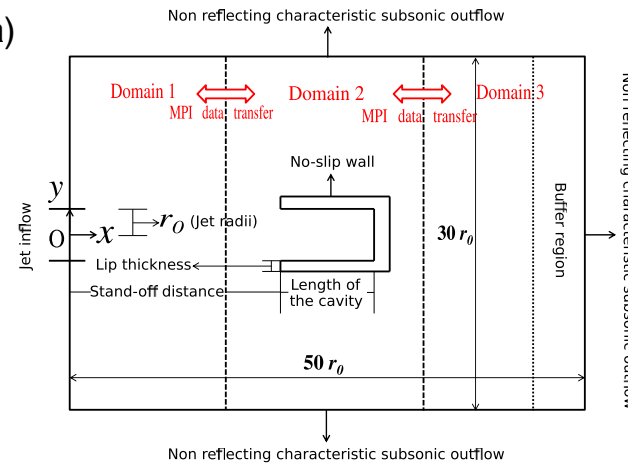

(c)

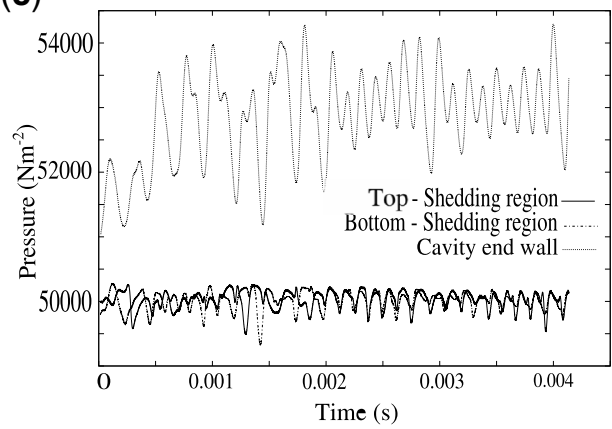

(b)

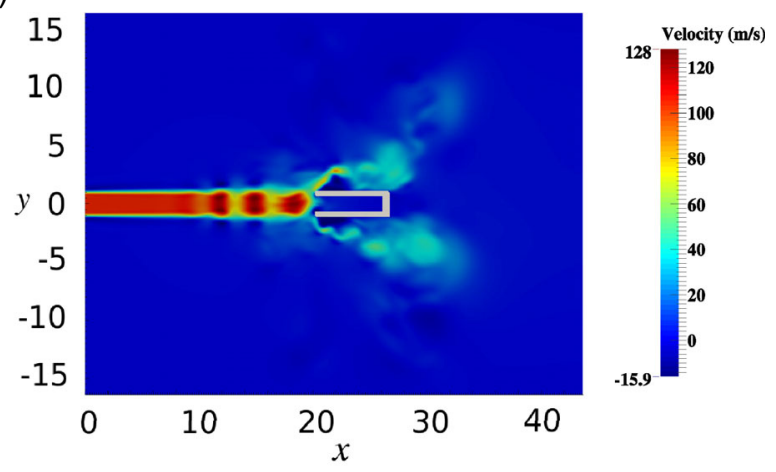

(d)

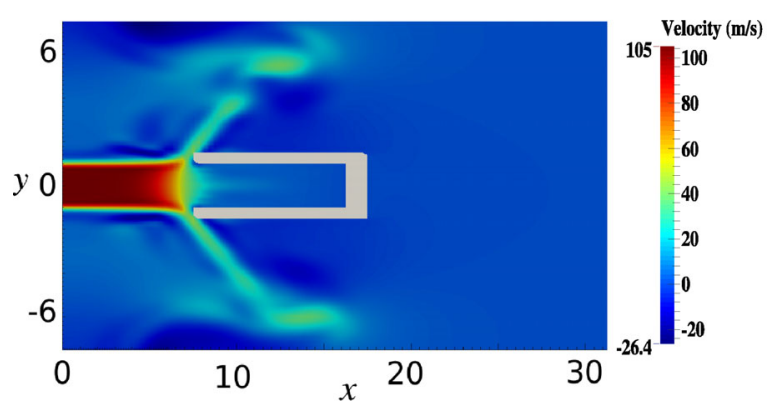

Figure 2. (a) Schematic of computational domain on the plane $z=0$, showing boundary conditions, axis, nomenclature and interface communication zones. (b) Axial velocity field at an instant on midplane $z=0$. (c) Pressure time history for simulation S1T1. (d) Axial velocity field at an instant on midplane $z=0$ (zoomed) for simulation S2T1.

Table 1. Dimensions of square cross-section tubes. All dimensions are scaled with jet radius $r_{j}$.

\begin{tabular}{lccccccc}
\hline \multirow{2}{*}{$\begin{array}{l}\text { Tube } \\
\text { type }\end{array}$} & \multicolumn{2}{c}{ Dimensions of outer wall } & & \multicolumn{2}{c}{ Dimensions of inner wall } \\
\cline { 2 - 3 } \cline { 6 - 8 } & Length & Breadth & Width & & Length & Breadth & Width \\
\hline T1 & 4.25 & 2.125 & 2.125 & & 4 & 2.0 & 2.0 \\
T2 & 4.25 & 2.250 & 2.250 & & 4 & 2.0 & 2.0 \\
T3 & 4.25 & 2.750 & 2.750 & & 4 & 2.5 & 2.5 \\
\hline
\end{tabular}

Table 2. Configurations of tube simulations.

\begin{tabular}{lcc}
\hline Simulation cases & $S$ & Tube type \\
\hline S1T1 & 20 & T1 \\
S1T2 & 20 & T2 \\
S2T1 & 7.5 & T1 \\
S2T3 & 7.5 & T3 \\
\hline
\end{tabular}

of the oscillations is quite small compared with the power of oscillations achieved by placing the tube in supersonic flow field. For tubes placed in supersonic jets, Narayanan et al [27] measured the maximum power to be on the order of $10^{6} \mathrm{~Pa}^{2} / \mathrm{Hz}$, whereas for these subsonic flows, maximum power observed is on the order of $10^{3} \mathrm{~Pa}^{2} / \mathrm{Hz}$. The effect of the stand-off shock as a source of periodic forcing is stronger than that of the vortex rings.

To establish a causal connection between the jet's ring vortices and tube oscillations, coherence was calculated between the upper lip and tube end wall pressure signals. Coherence between two signals $(x(t), y(t))$, is a real-valued function in the range of [0-1] defined as

$$
C_{x y}(f)=\frac{\left|\phi_{x y}(f)\right|^{2}}{\phi_{x x}(f) \phi_{y y}(f)}
$$

where $\phi_{i j}, \phi_{i i}$ are cross-power spectral density and autopower spectral density, respectively. When $C_{x y}(f) \approx 1$ the signals are perfectly related, whereas $\mathrm{C}_{x y}(f) \approx 0$ when they are uncorrelated. Figure $3 \mathrm{c}$ shows the close connection (high coherence levels) between excitation at the lip and the end wall at the dominant frequency and for secondary modes for case S1T1. Results for case S1T2 are similar.

OASPL curves along the line $y=6, z=0$ have been plotted in figure $3 \mathrm{~d}$. On this line, the levels of $150-165 \mathrm{~dB}$ are quite large. Even in a subsonic jet, the Hartmann whistle is a significant sound source. The minimum is obtained at sideline angles from the tube lip. Sound produced with the thicker tube (S1T2) is generally higher everywhere, slightly more upstream of the jet than downstream. Peak levels occur at about $22^{\circ}$ upstream and $13^{\circ}$ downstream with respect to the tube lip. Narayanan et al 
(a)

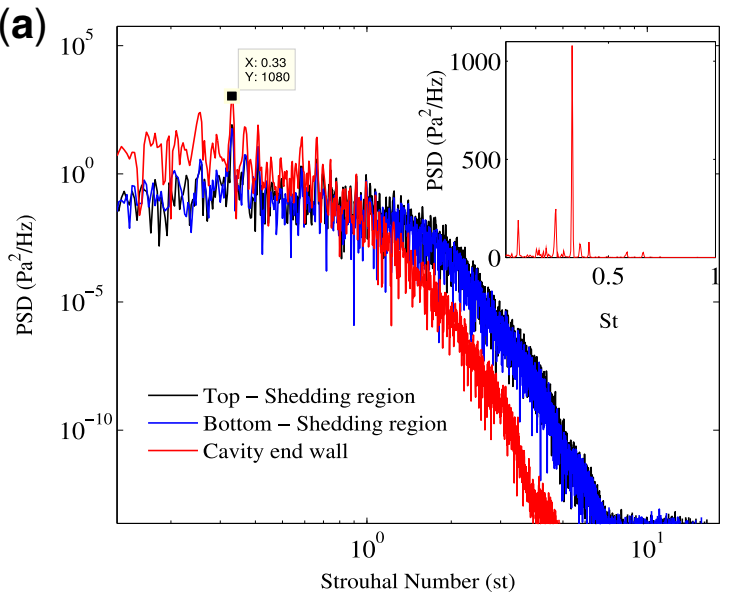

(c)

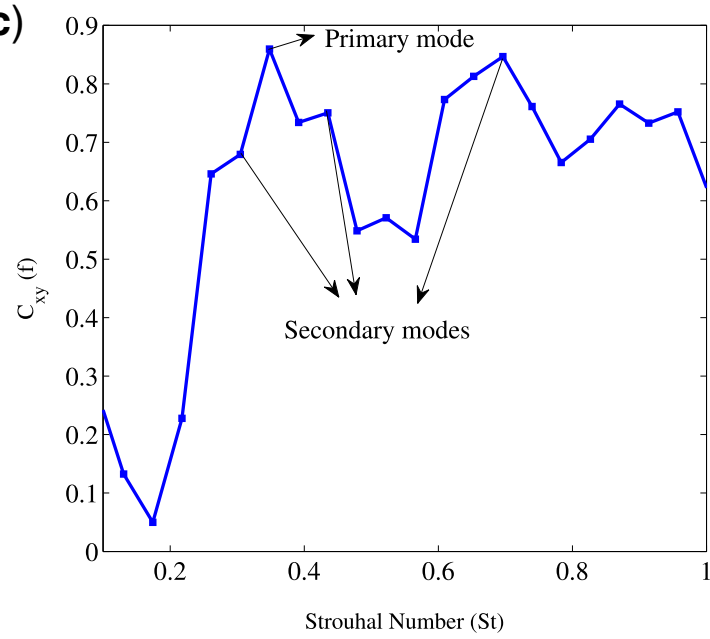

(b)

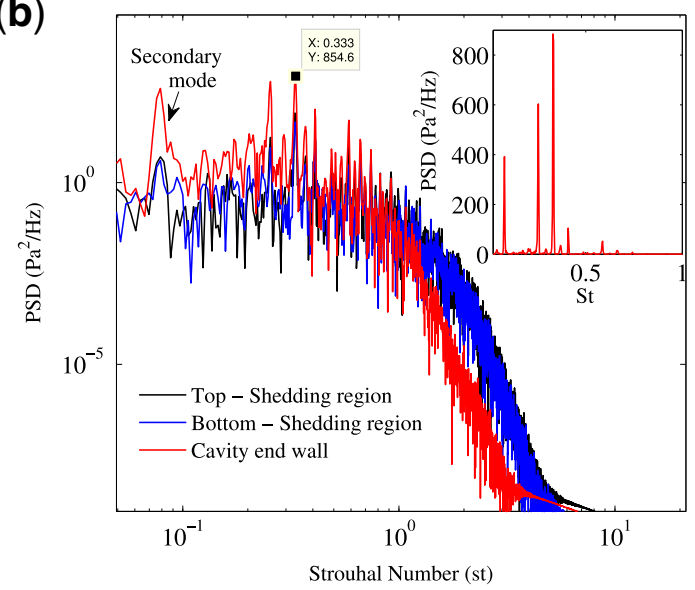

(d)

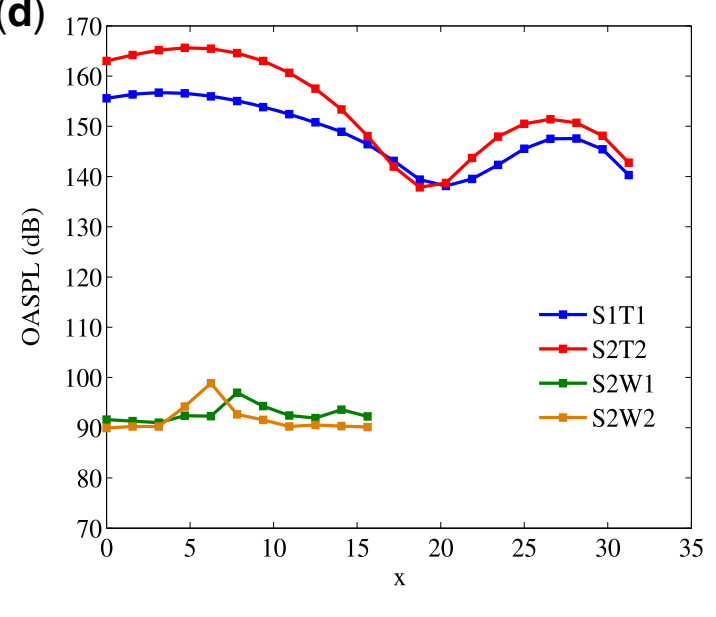

Figure 3. Frequency characteristics comparison: (a) lip thickness, $0.125 r_{0}$, (b) lip thickness, $0.250 r_{0}$ and (c) coherence analysis for simulation S1T1. (d) Overall sound pressure level (OASPL).

[27] showed that directivity of Hartmann whistle is found to be around $35^{\circ}-40^{\circ}$ for cavities placed in supersonic jet flow.

\subsection{Tube in potential core region}

In the second configuration, the open end of the tube was at $x=7.5$. Here, the annular shear layer has fluctuations responding to the forcing in the inflow plane but does not roll up into vortex rings. Figure $2 \mathrm{~d}$ is a visualization at an instant with contours of the velocity magnitude. The jet is deflected outward by the tube and the jet boundary breaks up. Within the tube there are pressure fluctuations, but of very small amplitue. OASPL along $y=6, z=0$ for the two cases S2T1 and S2T3 are shown in figure 3d. Clearly there has been no significant resonant response, even though the fundamental mode of the tube was matched with the dominant shear layer frequency. Increasing tube width (S2T3 simulations) did not change the response. Hence, the excitation by an unsteady shear layer is not sufficient to evoke a resonant response. The periodic impingement of the vortex rings seems to be crucial.

\section{Discussion and conclusions}

A computational study of a Hartmann whistle arrangement in a low subsonic round jet was conducted. The length of the tube was selected such that its fundamental duct mode was close to the dominant mode in the impinging jet. It was found that even a subsonic jet can provoke a strong resonant response if the tube is excited by the regular train of vortex rings that result from the instability of the jet's bounding shear layer. At a distance of 3 diameters from the jet, the OASPL is $150-160 \mathrm{~dB}$. A thicker tube lip was found to support larger SPL due to increased response at frequencies lower than the fundamental. These lower frequencies were not subharmonics. Although the Hartmann whistle in a supersonic stream exhibits power levels that are $\mathrm{O}(1000)$ times larger, the response in a subsonic stream is not insignificant. If the tube is placed closer to the jet exit, where the jet shear layer is unsteady but has not broken up into vortex rings, the excitation does not provoke any significant response at all. A direct comparison of these computational results with other published results is not 
feasible due to the lack of any other experimental or computational studies in subsonic regime. However, our simulations are qualitatively similar to simulations of an impulsive subsonic jet actuator [8], and experiments reviewed in the introduction $[9,10]$.

The resonant response provoked by the train of vortex rings demonstrates that the actions of stems or wires on the axis are due to the vortex shedding that they produce. In this case, these impinging vortices were generated without such devices as the end result of shear layer instability.

\section{Acknowledgements}

We thank Dr Santosh Hemachandra, Aerospace Engineering, IISc, for helpful discussions of this work.

\section{List of symbols}

A

$a$

f

$p$
$L$

$M$

$p_{0}$

$R$

$\operatorname{Re}$

$r_{j}$

$S$

St

$U_{j}$

$x$

$x_{i n}$

$x_{\infty}$

$\rho$

$\theta$

$\phi_{i j}(f)$

$\phi_{i i}(f)$

$\tau_{i j}$

$\mu$

PSD

SPL

OASPL

\author{
area of the tube inlet $\left(\mathrm{m}^{2}\right)$ \\ speed of sound $\left(\mathrm{ms}^{-1}\right)$ \\ frequency $(\mathrm{Hz})$ \\ length of the tube $(\mathrm{m})$ \\ Mach number \\ static pressure $\left(\mathrm{N} / \mathrm{m}^{2}\right)$ \\ total pressure $\left(\mathrm{N} / \mathrm{m}^{2}\right)$ \\ nozzle pressure ratio $\left(p_{0} / p_{\infty}\right)$ \\ Reynolds number \\ jet radius $(\mathrm{m})$ \\ stand-off distance $(\mathrm{m})$ \\ Strouhal number $\left(f U_{j} / 2 r_{0}\right)$ \\ jet velocity at nozzle exit $\left(\mathrm{ms}^{-1}\right)$ \\ reduced length (no unit) \\ value of variable $x$ at nozzle exit \\ value of variable $x$ at ambient conditions \\ density $\left(\mathrm{kg} / \mathrm{m}^{3}\right)$ \\ polar angle (degrees) \\ cross-power spectral density $\left(\mathrm{Pa}^{2} / \mathrm{Hz}\right)$ \\ auto-power spectral density $\left(\mathrm{Pa}^{2} / \mathrm{Hz}\right)$ \\ shear stress tensor \\ dynamic viscosity $\left(\mathrm{Nsm}^{-2}\right)$ \\ power spectral density $\left(\mathrm{Pa}^{2} / \mathrm{Hz}\right)$ \\ sound pressure level $(\mathrm{dB}), \mathrm{p}_{\text {ref }}=20 \times 10^{-6} \mathrm{~Pa}$ \\ overall sound pressure level $(\mathrm{dB})$
}

\section{References}

[1] Hartmann J 1922 On a new method for the generation of sound-waves. Phys. Rev. 20(6): 719

[2] Raman G and Srinivasan K 2009 The powered resonance tube: from Hartmann's discovery to current active flow control applications. Prog. Aerosp. Sci. 45(4): 97-123

[3] Chang K S, Kim K H and Iwamoto J 1996 A study on the Hartmann-Sprenger tube flow driven by a sonic jet. A study on the Hartmann-Sprenger tube flow driven by a sonic jet 13(3): 173-182

[4] Hamed A, Das K and Basu D 2002 Numerical simulation of unsteady flow in resonance tube. AIAA Paper 1118:2002

[5] Cain A B, Kerschen E J and Raman G 2002 Simulation of acoustic characteristics and mechanisms of powered resonance tubes. In: Proceedings of the 8th AIAA/CEAS Aeroacoustics Conference and Exhibit, Breckenridge, $C O$

[6] Hamed A, Das K and Basu D 2003 Numerical simulation and parametric study of Hartmann-Sprenger tube based powered device. In: Proceedings of the 41st Aerospace Sciences Meeting and Exhibit, Reno, NV, USA, pp. 6-9

[7] Hamed A, Das K, and Basu D 2003 Characterization of powered resonance tube for high frequency actuaton. FEDSM2003-45472

[8] Braud C and Dyment A 2012 Model of an impulsive subsonic jet actuator for flow control applications, Phys. Fluids 24(4): 047102

[9] Sprenger H 1954 On thermal effects in resonance tubes. Mitt. Inat. Aerodyn. ETH 21: 18-24

[10] Brocher E, Maresca C and Bournay M H 1970 Fluid dynamics of the resonance tube. J. Fluid Mech. 43:369-384

[11] Sarohia V and Back L H 1979 Experimental investigation of flow and heating in a resonance tube. J. Fluid Mech. 94: 649-672

[12] Brocher E and Duport E 1988 Resonance tubes in a subsonic flowfield. AIAA J. 26(5): 548-552

[13] Mathew J, Lechner R, Foysi H, Sesterhenn J and Friedrich R 2003 An explicit filtering method for large eddy simulation of compressible flows, Phys. Fluids 15(8):2279-2289

[14] Visbal M R and Rizzetta D P 2002 Large-eddy simulation on curvilinear grids using compact differencing and filtering Schemes, ASME J. Fluids Eng. 124(December): 836

[15] Bogey C and Bailly C 2006 Computation of a high Reynolds number jet and its radiated noise using large eddy simulation based on explicit filtering. Comput. Fluids 35(10): 1344-1358

[16] Hixon R and Turkel E 2000 Compact implicit MacCormacktype schemes with high accuracy. J. Comput. Phys. 158(1): 51-70

[17] Lele S K 1992 Compact finite difference schemes with spectral-like resolution. J. Comput. Phys. 103(1):16-42

[18] Poinsot T J and Lele S K 1992 Boundary conditions for direct simulations of compressible viscous flows. J. Comput. Phys., 101(1): 104-129

[19] Ganesh S 2016 Large eddy simulation of free and impinging subsonic jets and their sound fields. $\mathrm{PhD}$ Thesis, Department of Aerospace Engineering, Indian Institute of Science

[20] Bodony D J and Lele S K 2004 Jet noise prediction of cold and hot subsonic jets using large-eddy simulation. In: Proceedings of the 10th AIAA/CEAS Aeroacoustics Conference. Paper AIAA-2004-3022

[21] Bogey C and Bailly C 2006 Large eddy simulations of transitional round jets: influence of the Reynolds number on flow development and energy dissipation. Phys. Fluids 18(6): 065101

[22] Panchapakesan N R and Lumley J L 1993 Turbulence measurements in axisymmetric jets of air and helium. Part 1. Air jet. J. Fluid Mech. 246:197-223

[23] Zaman K B M Q 1998 Asymptotic spreading rate of initially compressible jets-experiment and analysis. Phys. Fluids 10(10): 2652 
[24] Varadharajan R 2016 LES study of free jets and jets impinging on cuboidal cavity. Master Thesis, Department of Aerospace Engineering, Indian Institute of Science

[25] Varadharajan R 2017 Reynolds number effects on transition, turbulence intensity and axial-velocity decay rate of turbulent round jets. arXiv preprint arXiv:1708.03140
[26] Freund J B 2001 Noise sources in a low-Reynolds-number turbulent jet at Mach 0.9. J. Fluid Mech. 438: 277-305

[27] Narayanan S, Bhave P, Srinivasan K, Ramamurthi K and Sundararajan T 2009 Spectra and directivity of a Hartmann whistle, J. Sound Vib. 321(3): 875-892 